\title{
Diagnóstico de la actividad pesquera artesanal en la Bahía de Tela
}

\author{
Ernesto Alcides Carbajal Marquez ${ }^{1}$ \\ Liliana Paola Sierra Castillo ${ }^{2}$ \\ Ester Agar López Irías ${ }^{3}$
}

\section{RESUMEN}

El siguiente artículo describe las características que presenta la pesca artesanal en la Bahía de Tela, esta actividad es una de las más significativas para las comunidades del área, no solo por las ventajas que implica realizar esta activad (independencia, nutrición y seguridad alimentaria) pero también, debido a la falta de alternativas económicas y empleo, la pesca es una fuente de ingreso permanente para los pescadores. El objetivo general fue el de elaborar herramientas de manejo y conservación pesquera para la Bahía de Tela (costa norte de Honduras), incluyendo el Refugio de Vida Silvestre Marino Bahía de Tela, Parque Nacional Punta Izopo y Parque Nacional Jeannette Kawas. Para el análisis de la situación socioeconómica de las comunidades pesqueras, se emplearon distintas metodologías como los siguientes: censos pesqueros y encuestas socioeconómicas, esto permitió conocer la problemática que enfrentan.

Para los muestreos biológicos, se consideraron tres comunidades representativas del área: las comunidades garífunas de Tornabe, Miami y Tela. La toma de datos de los muestreos pesqueros abarca el periodo de tiempo comprendido desde febrero del 2015- abril del 2016. Con el fin de evaluar los recursos pesqueros se tomaron consideraciones ecológicas, dinámica de poblaciones y esfuerzo pesquero. La pesquería en Tela se encuentra en un estado crucial, ya que aún hay un porcentaje de especies que pertenecen a niveles altos en la cadena trófica; sin embargo si se sigue explotando insosteniblemente, aumentará la explotación de carnívoros de primer orden y luego herbívoros; afectando la salud del ecosistema y la pesquería.

Palabras clave: pesca artesanal, recursos pesqueros, muestreos biológicos, nivel de

\footnotetext{
${ }^{1}$ Estudiante, de la Carrera de Biología, Escuela de Biología, Facultad de Ciencias, UNAH. Correo electrónico: ernestocarbajalm@gmail.com.

${ }^{2}$ Licenciada en Biología, CORAL Reef Alliance, correo electrónico: lilianap.sierra@gmail.com.

${ }^{3}$ Profesora universitaria y asesora, Escuela de Biología, Facultad de Ciencias, UNAH. Correo electrónico: ester.lopez@unah.edu.hn.
} 


\section{ABSTRACT}

The following article details the principle characteristics that the artisanal fisheries of the Bay of Tela present. This activity is one of the most significant for the communities in the area for the great advantages that it presents (independence, nutrition and food security) and because of the lack of sources of income, fishing is a permanent source of income for fishermen and their families. The main objective was to elaborate fishing management and conservation tools for the Bay of Tela, including the Site of Wildlife Importance Bay of Tela , the National Park Punta Izopo and the National Park Jeannette Kawas. For the socieconomic analyses of the fisheries communities various methodologies were employed, such as: fisheries census and socioeconomic surveys, this tools allowed to understand the current problematic that this community's face.

For the biological samples, three representative communities of the area were considered: the garifuna communities of Tornabe, Miami and Tela. The data collection was done in the time period of time between February 2015- Abril 2016. With the purpose of evaluating the fishery resources, ecological considerations, population dynamics and fishing effort were considered. The artisanal fisheries in Tela are in a crucial state, this is because there is still a percentage of species that belong to high levels of the trophic chain, however if the fishery resources are continued to be exploited, the exploitation of first order carnivores and herbivores will increase, affecting the ecosystem's health and the fishery.

Keywords: Artisanal fisheries, Fishery Resources, Biological Sampling, Exploitation Level, Marine Protected Areas. 


\section{INTRODUCCIÓN}

La región del Caribe está dotada con una gran riqueza de recursos marinos y costeros, incluyendo una maravillosa multitud de plantas y animales únicos (Burke \& Maidens, 2005). Esta riqueza ha hecho que los recursos marinos y costeros sean uno de los más explotados de los últimos tiempos, según (FAO, 1996) la explotación de los recursos costeros y de sus hábitat está en función del tamaño de la población humana y de su grado de desarrollo socioeconómico, el constante crecimiento demográfico, y su concentración acelerada en zonas costeras y centros urbanos costeros. El suministro de peces comestibles se ha incrementado a una tasa media anual del $3,2 \%$, superando así la tasa de crecimiento de la población mundial del 1,6\%. El consumo aparente mundial de pescado per cápita aumentó de un promedio de $9,9 \mathrm{~kg}$ en el decenio de 1960 a 19,2 kg en 2012, según las estimaciones preliminares. Este incremento notable se ha debido a una combinación de crecimiento demográfico, aumento de los ingresos y urbanización, y se ha visto propiciado por la fuerte expansión de la producción pesquera y la mayor eficacia de los canales de distribución (FAO, 2014).

La sobreexplotación pesquera es una amenaza generalizada en el arrecife mesoamericano y, debido a la alta biodiversidad en los ecosistemas coralinos es difícil predecir las alteraciones resultado de la extracción de un gran número de especies por parte de los pescadores (WWF, 2006). Según Burke \& Maidens (2005) en la actualidad, los arrecifes coralinos son extremadamente importantes para las economías de los países del Caribe y son la reserva de capital para la futura seguridad económica y política, y Tela no es la excepción.

Según estudios realizados por científicos, Tela cuenta con el segundo arrecife con mayor cobertura de coral vivo en el Sistema Arrecifal Mesoamericano. Debido a la importancia ecológica y socioeconómica que representa el arrecife de coral para las comunidades costeras de la Bahía de Tela , se desarrolló el presente estudio biológico y socioeconómico de la pesquería artesanal en Tela, con el fin de lograr el aprovechamiento sostenible, elaborando herramientas de manejo y conservación pesquera de manera conjunta entre la academia (UNAH) a través de dos pasantes de práctica profesional supervisada con la asesoría de una docente de la Escuela de Biología, pescadores de las distintas comunidades, Coral Reef Alliance (CORAL), Fondo Mundial para la Naturaleza (WWF), DIGEPESCA, ICF y PROLANSATE.

De esta alianza estratégica surge el proyecto de vinculación "Elaboración de herra- 
mientas de manejo y conservación pesquera para la Bahía de Tela, incluyendo el Refugio de Vida Silvestre Marino Bahía de Tela, Parque Nacional Punta Izopo y Parque Nacional Jeannette Kawas" cuyo propósito fue caracterizar la actividad pesquera de la Bahía de Tela, ya que es fuente de nutrición, ingresos económicos y bienestar en la comunidades.

\section{MÉTODO}

Para lograr el análisis de la situación socioeconómica de las 13 comunidades pesqueras de la Bahía de Tela se emplearon varios métodos, los cuales se mencionan a continuación. Se realizaron censos pesqueros en el año 2015 en conjunto con el Fondo Mundial para la Naturaleza (WWF), Universidad Nacional Autónoma de Honduras (UNAH) y CORAL, esto permitió contar con una línea base y conocimiento sobre las comunidades y la problemática que presentan. Igualmente se realizaron múltiples encuestas socioeconómicas y conversatorios comunitarios con los pescadores y sus familias para obtener información socioeconómica real y actualizada. Para lograr validar la información colectada se llevó a cabo una serie de diagnósticos comunitarios con todos los pescadores (organizados y no organizados) de las distintas comunidades pesqueras.

Para la evaluación de los recursos pesqueros se aplicó el método clásico basado en la dinámica de poblaciones (Sparre \& Venema, 1997), se trata de un método analítico que utiliza la distribución de frecuencia de longitudes de los organismos. En este método el primer paso es la estimación de la mortalidad total (Z) a partir de la curva de captura linealizada, para el posterior análisis de rendimiento máximo sostenible (RMS), este último obtenido con el análisis de cohorte de Jones (Sparre \& Venema, 1997). Para la estimación del valor Mortalidad Total (Z), se utilizó la siguiente fórmula:

$$
\frac{\ln C(L 1 . L 2)}{\Delta t(L 1, L 2)}=c-Z * t \frac{L 1+l 2}{2}
$$

Donde In C (L1.L2) logaritmo natural de la captura, $\Delta t$ (L1.L2) es el tiempo que tarda el organismo en crecer de la talla 1 (L1) a la talla 2 (L2). Este es una ecuación líneal donde la pendiente equivale a la mortalidad total (Sparre \& Venema, 1997). 
Para el anterior análisis, se efectuaron muestreos biológicos para los cuales se consideraron tres comunidades representativas del área: las comunidades garífunas de Tornabe, Miami y todos los pescadores de las colonias y barrios del centro de Tela. La toma de datos de los muestreos biológicos abarca el periodo de tiempo comprendido desde febrero del 2015- abril del 2016. La unidad de muestreo pesquero fue la embarcación de pesca cuando regresaba de faenar en cada comunidad seleccionada, la misma era elegida al azar, al momento del arribo de varias embarcaciones. Para cada especie comercial capturada en los desembarques, se determinó el nombre común (el nombre conocido coloquialmente por los pescadores de las comunidades), género y especie (Cervigón, et al., 1992), la longitud de horquilla en centímetros (LH) con un ictiometro y peso en gramos de cada individuo con una balanza analítica; en algunos casos estadio gonadal, esta información era introducida sistemáticamente en una base de datos para los posteriores análisis correspondientes como estructura de talla multiespecifica, distribución de frecuencia de talla por especie y por arte de pesca (Gulland \& Rosenberg, 1992). Comparación entre las tallas de primera captura recomendada y observada (bibliográficamente y datos gonadales de los muestreos). Sumado a esta información se obtuvo características de las zonas de pesca, artes de pesca y esfuerzo de pesca (Horas, días). Al mismo se consideró el enfoque Ecosistémico, (FAO, 2010), tomando datos ecológicos como el hábitat y nivel trófico de los organismos en su etapa adulta.

Para la realización de los muestreos biológicos y encuestas pesqueras se contó con colaboración de los pescadores y sus familias, se recalcó en todo momento la confidencialidad de los datos y su tratamiento a nivel global y no personal. Los resultados del estudio fueron socializados con los pescadores artesanales, actores locales y autoridades.

\section{RESULTADOS Y DISCUSIÓN}

\section{Aspectos sociales y económicos de las distintas comunidades pesqueras de Tela}

Entre las actividades económicas de la Bahía de Tela se puede mencionar, el monocultivo de palma africana, remesas y la pesca. Ante la falta de alternativas de empleo se puede evidenciar que la pesca es una de las actividades económicas de mayor relevancia y de mayor accesibilidad a las comunidades más vulnerables. Se estima un total de 725 pescadores activos, de estos un aproximado de 440 pescan exclusi- 
vamente en la Laguna de Los Micos, 560 alternan su pesca entre la laguna y el mar, finalmente 165 pescadores realizan dicha actividad únicamente en el mar. Un aproximados de 60 días al año se consideran días de no pesca, determinado por la condiciones climáticas y afectando todas las faenas de pesca con las distintas artes en el mar, en cambio el esfuerzo de pesca en la laguna es más estable debido a la geomorfología que esta posee.

Es importante mencionar, que las comunidades que pescan en la Laguna de los Micos, extraen peces y jaiba para comercializar y como subsistencia. Las comunidades de Los Cerritos y Marion, capturan peces, jaiba y en algunas temporadas extraen almejas. La comunidad de Las Tuzas se dedica casi en su exclusividad a la extracción de jaibas.

De la misma manera, la mujer tiene un rol fundamental en la actividad pesquera en las distintas comunidades estudiadas. En comunidades como Marion, Los Cerritos y Agua Chiquita las mujeres participan de manera activa en la extracción de producto pesquero y en la comercialización del mismo (Figura 1).

Figura 1. Mujeres pescadoras de la comunidad de Marion.

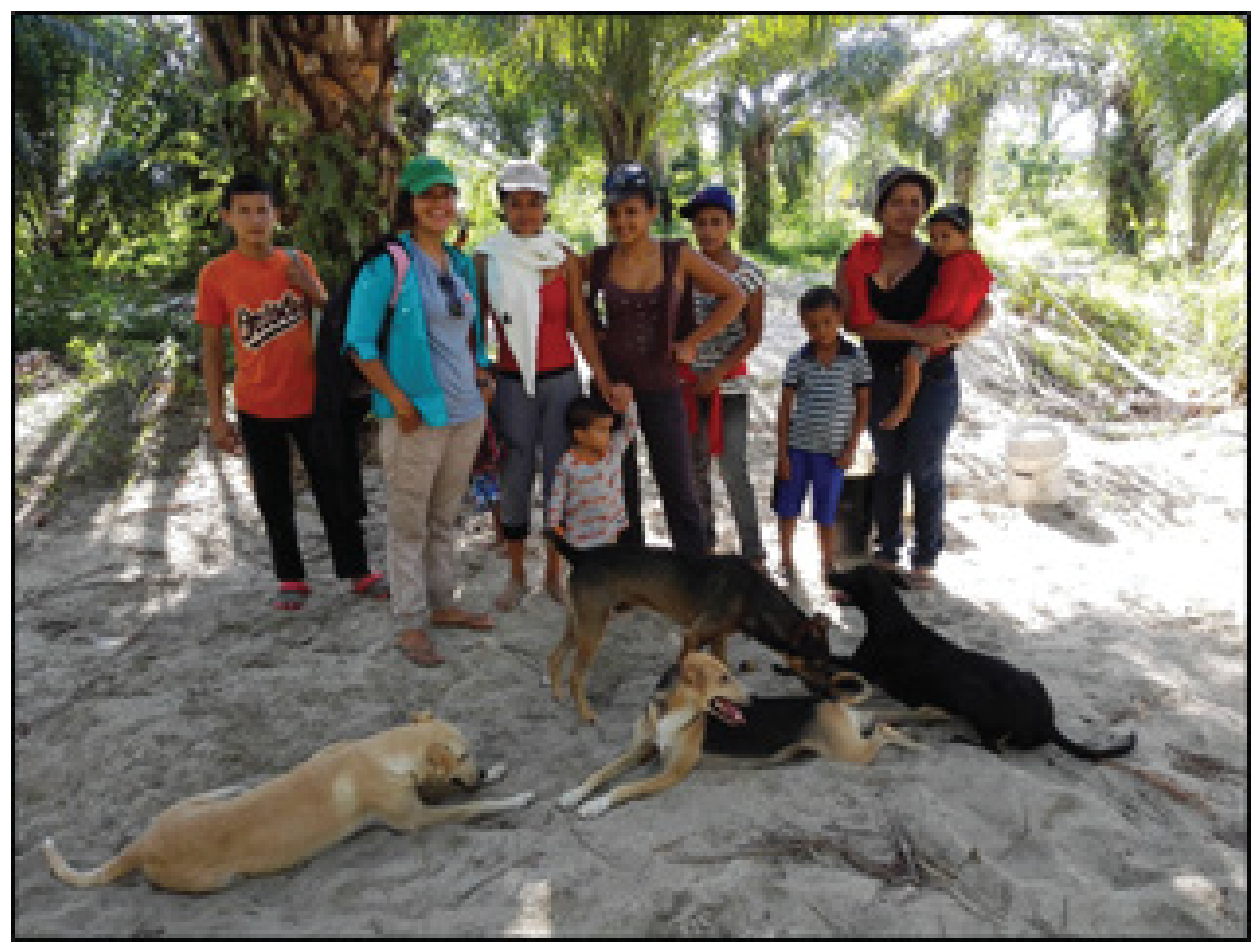

Fuente: fotografía Ernesto Carbajal 
Paralelamente, en las comunidades garífunas, la mujer es clave para la comercialización del producto (ella es la encargada de comprar y vender el producto pesquero).

Según la comunidad y la zona de pesca que es utilizada, las artes de pesca empleadas varían. Las comunidades que residen alrededor de la Laguna de Los Micos y que pescan en dicha laguna utilizan en su mayoría redes agalleras, conocidas entre los pescadores como trasmallos (Figura 2), similarmente utilizan los netes (40 netes por pescador) para la captura de jaibas (Figura 3), arpones y en una menor proporción la línea y anzuelo. Las comunidades garífunas que pescan en el mar (San Juan, Triunfo de La Cruz, Tornabe, La Ensenada, Miami y Barra Vieja) pescan en su mayoría utilizando el chinchorro, ya que es considerado una manera de pescar tradicional y forma parte de su cultura; similarmente utilizan redes agalleras, línea y anzuelos, nasas (trampas) para peces y arpones.

Figura 2. Pescador limpiando sus redes agalleras.

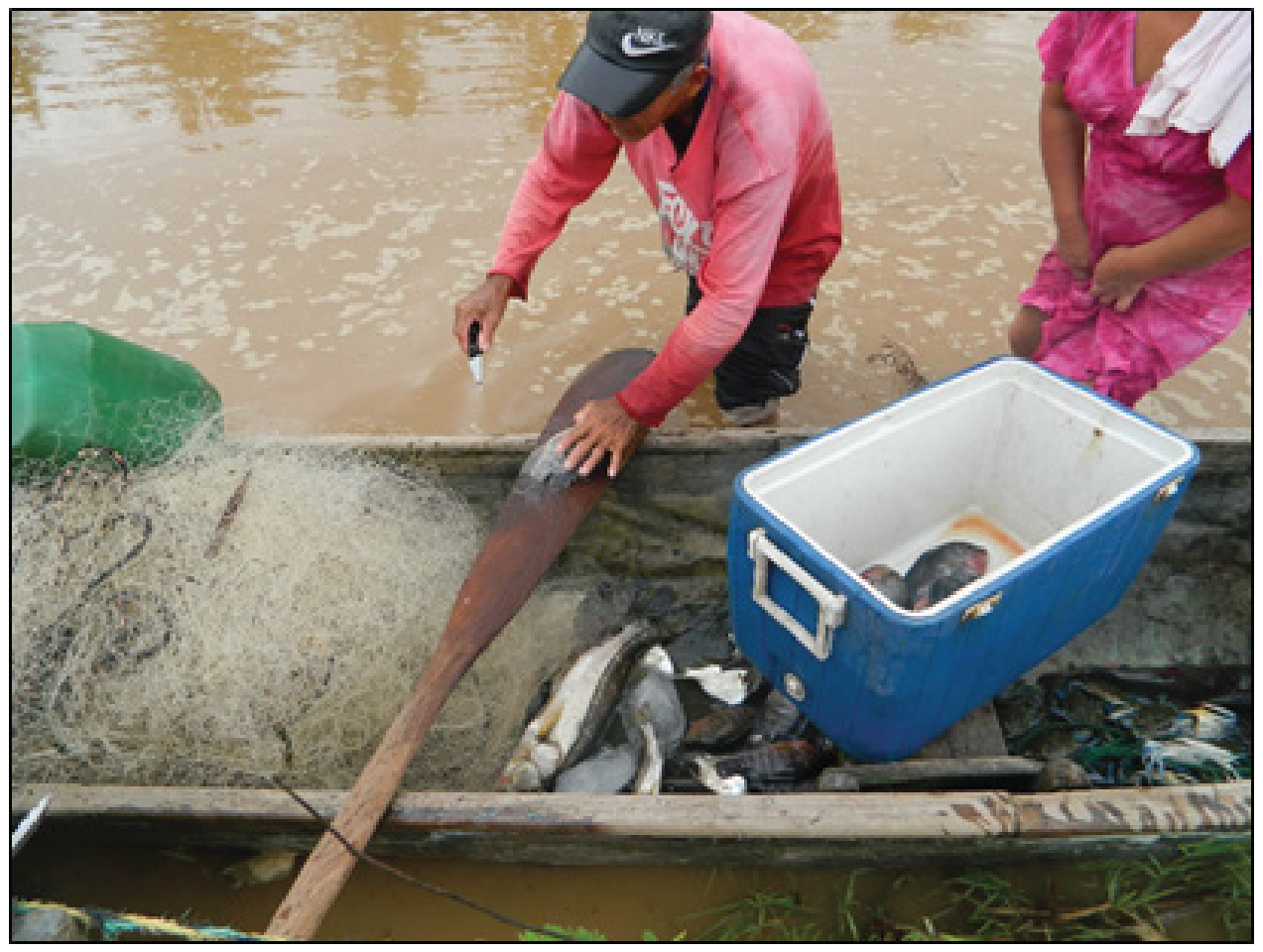

Fuente: fotografía Ernesto Carbajal 
Figura 3. Netes utilizados por pescadores para la captura de jaibas.

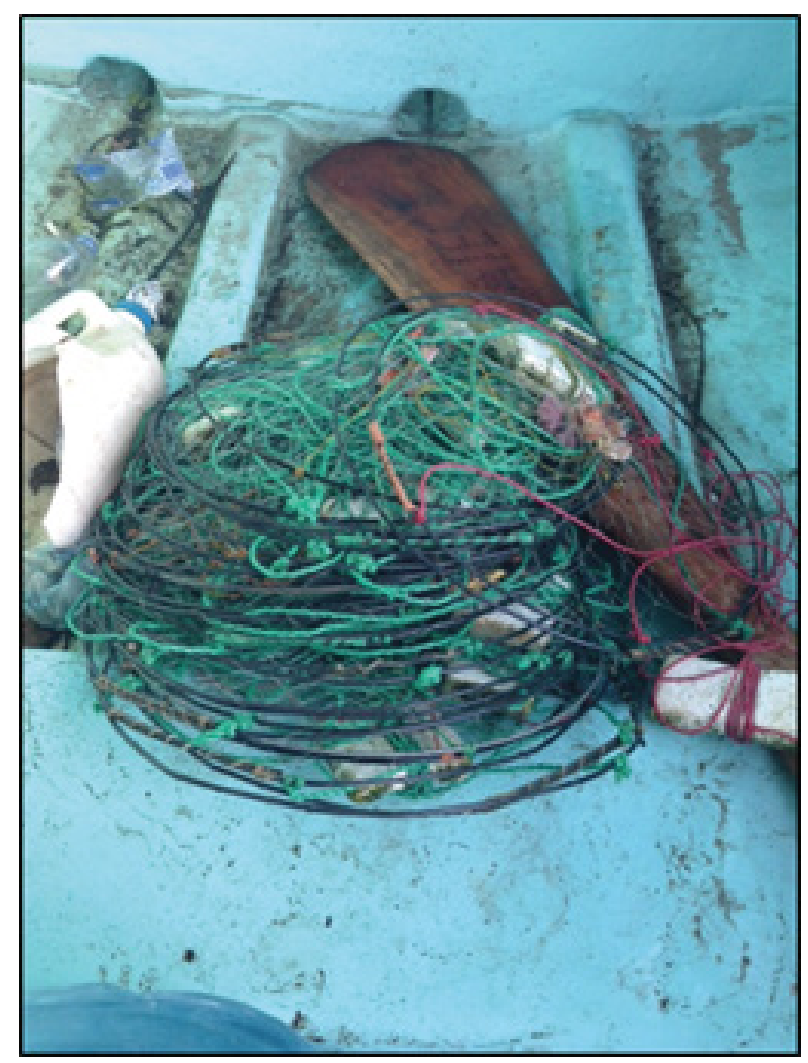

Fuente: fotografía Ernesto Carbajal

\section{Evaluación de los recursos pesqueros}

En los muestreos biológicos realizados en las comunidades de Miami, Tornabe y Tela se llegó se logró identificar un total de 38 especies de peces, de los cuales la gran mayoría son especies de importancia comercial en el caribe hondureño como los pertenecientes a la familia Lutjanidae (pargos), Scombridae (macarelas), Carangidae (jureles) y Centropomidae (robalo), los individuos pertenecientes a esta última familia fueron capturados en sistemas lagunares.

Al respecto la información sobre la captura anual en libras de la zona costera, que comprende desde Omoa a Triunfo de la Cruz señala que, las dos principales familias de peces son Lutjanidae y Carangidae (PROLANSATE, 2000), si estos registros anteriores se comparan con el estudio actual, se puede apreciar que familia Lutjanidae 
(pargos) mantiene el primer lugar en la pesca, en cambio la familia Carangidae (jureles) se ve desplazada por la familia Scombridae (macarelas).

Se logró muestrear un total de 4031 individuos correspondientes a 38 especies comprendidas en 18 familias, siendo Lutjanus synagris (Figura 4) la especie más representativa con un total de 1815 individuos es decir un $45 \%$ de los peces medidos, seguido por Caranx crysos (Figura 5) con 641 individuos es decir un $16 \%$ y la macarela (Scomberomorus maculatus) (Figura 6) con un 7\%, como se observa en el gráfico 1.

Figura 4. Calale (Lutjanus synagris).

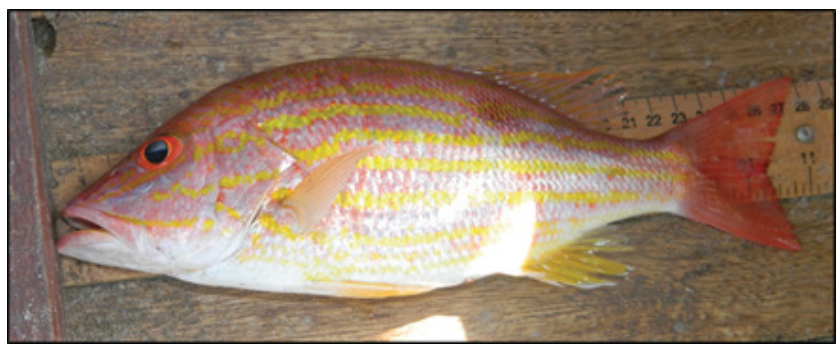

Fuente: fotografía Liliana Sierra

Figura 5. Culila (Caranx crysos).

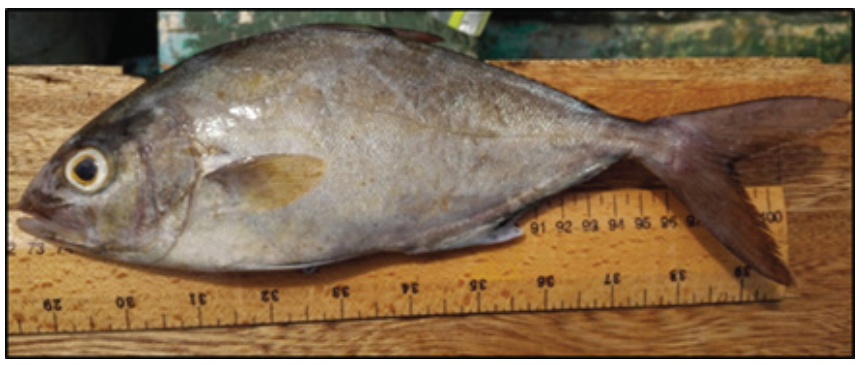

Fuente: fotografía Liliana Sierra

Figura 6. Macarela (Scomberomorus maculatus).

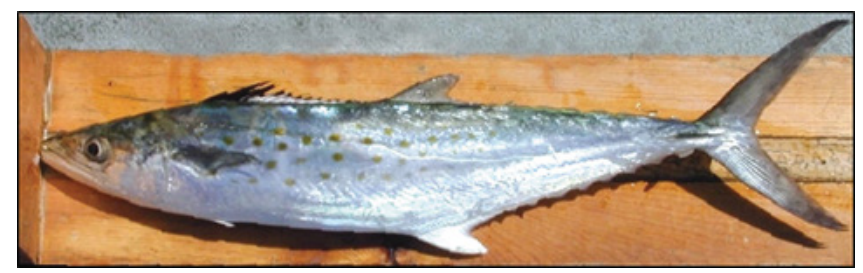

Fuente: fotografía Liliana Sierra 


\section{Gráfico 1. Principales especies de peces encontradas.}

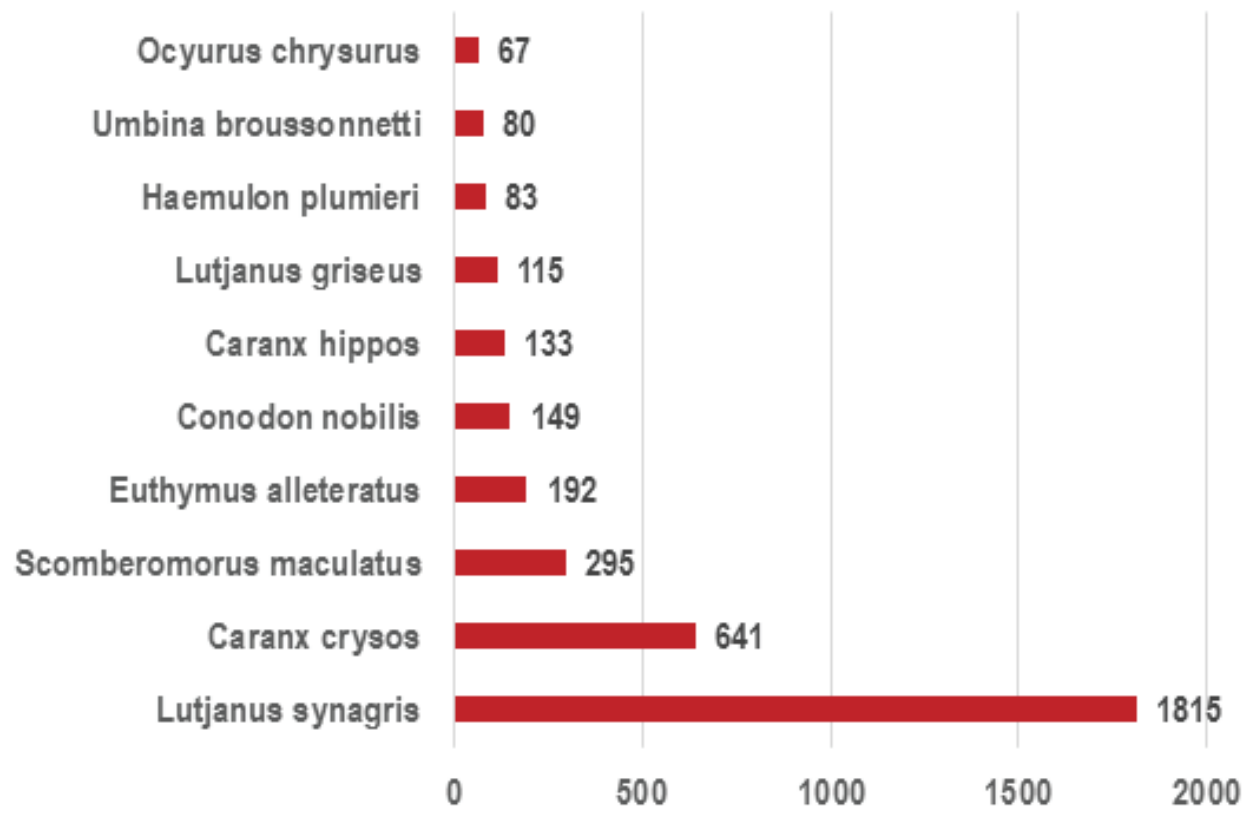

Fuente: muestreos biológicos

En cuanto al hábitat, de las 38 especies de peces identificadas, el $74 \%$ son especies arrecifales 0 asociadas al arrecife, el 17\% son especies pelágicas costeras, un $6 \%$ son especies demersales costeras.

De las 38 especies reportadas por los pescadores y encontradas en los muestreos biológicos, aproximadamente un $80 \%$ son especies que en alguna etapa de su ciclo de vida necesitan de las lagunas costeras para sobrevivir, por lo que destaca su importancia en procesos ecológicos como la conectividad.

Una de las relaciones más descritas en ecología y que vincula a las especies que habitan dentro de un sistema en particular es la alimentación, a través de las redes tróficas, lo que constituye la búsqueda de los regímenes alimenticios de cada especie (López, 2008). De las 38 especies reportadas en los muestreos biológicos, un 84\% son carnívoros de segundo orden (ingieren invertebrados bentónicos y añaden peces en su dieta), un $12 \%$ son piscívoros y un $2 \%$ son carnívoros de primer orden (se alimentan principalmente de invertebrados bentónicos). 
El Lutjanus synagris (calale) constituye la especie más importante, para la cual es posible indicar que, la talla media de captura es de 21 centímetros, sin embargo la talla media de maduración sexual (obtenida a través de muestras gonadales y estudios bibliográficos para la zona del mar caribe) es de 23 centímetros (grafico 2). Las tallas adecuadas para mantener el recurso pesquero deben superar la talla media de maduración sexual de la población de los organismos.

\section{Gráfico 2. Distribución de frecuencia de tallas para el calale, redes de 2".}

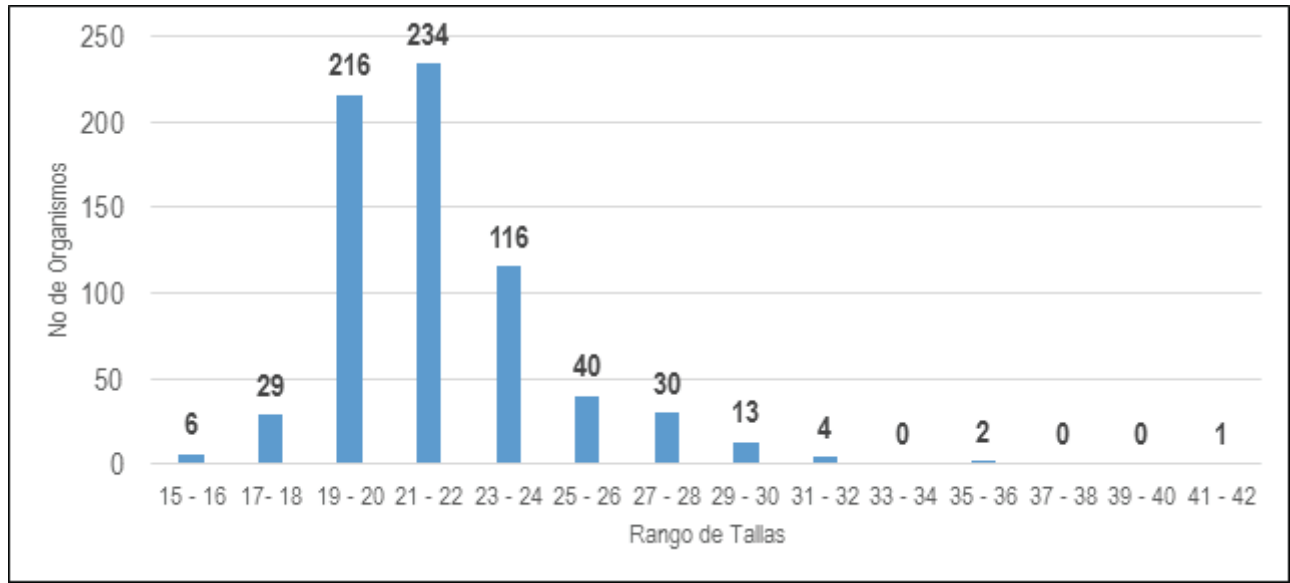

Fuente: muestreos biológicos

Mortalidad Total Z para Lutjanus Synagris, redes de 2 pulgadas (2 "):

Una "cohorte" es un grupo de peces de aproximadamente la misma edad pertenecientes a la misma población. La mortalidad de una cohorte (Z) se compone de la mortalidad provocada por la pesca $(F)$ y la mortalidad natural $(M)$, que comprende las muertes por depredación, enfermedad y muerte por edad avanzada (Sparre \& Venema, 1997).

Con la distribución de la frecuencia de tallas y la utilización de las constantes recomendadas por la bibliografía próximas a la región: $L$ infinito $=54 \mathrm{cms}$ y $K=0.51$, se logró obtener la estimación de la mortalidad total Z, mediante la curva de captura linealizada basada en tallas (gráfico 3).

El valor de la mortalidad total (Z) fue de 0.9 (límite de confianza de $95 \%$ ), este valor indica una tasa de explotación mediana, tal y como se aprecia en el cuadro 1. 
Gráfico 3. Curva de captura linealizada para Lutjanus synagris (calale) redes de 2".

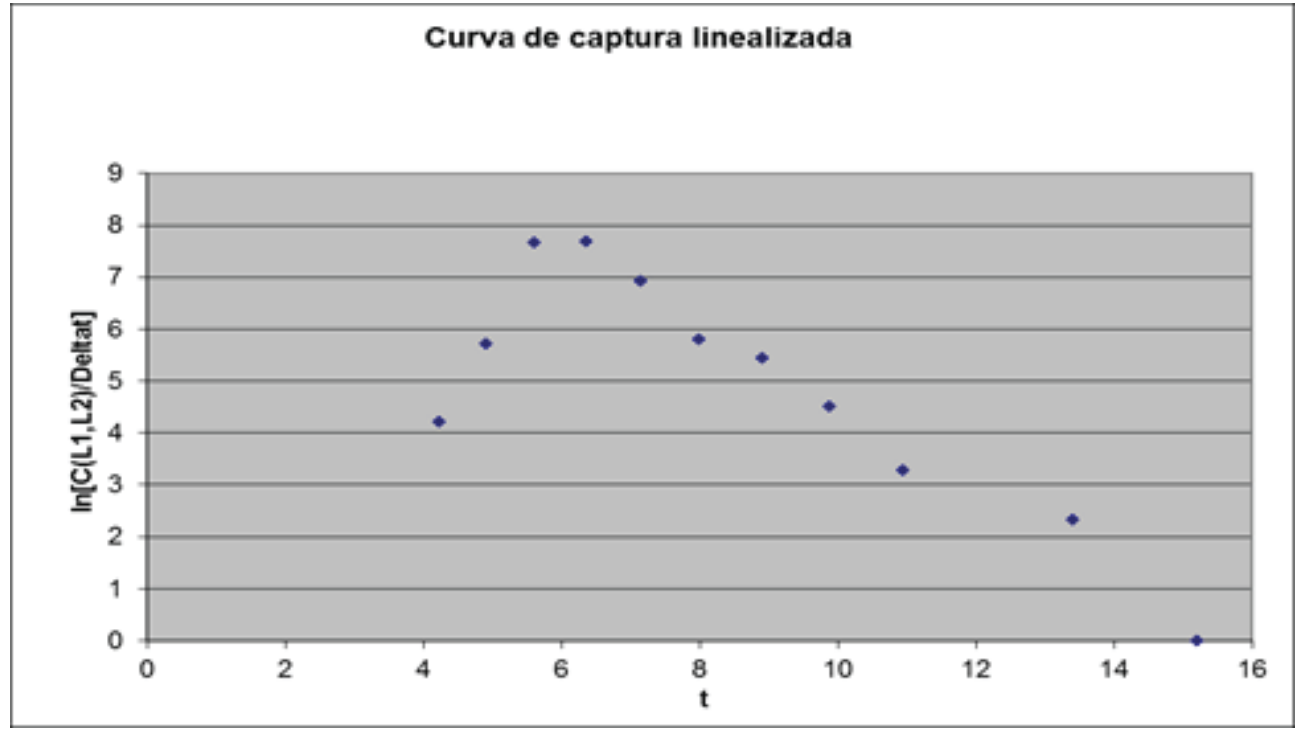

Fuente: muestreos biológicos

Cuadro 1. Niveles de explotación pesquera.

\begin{tabular}{|l|l|}
\hline \multicolumn{1}{|c|}{ Valor de z por año } & \multicolumn{1}{c|}{ Grado de explotación } \\
\hline 0.6/año & Explotación leve \\
\hline 0.9/año & Explotación mediana \\
\hline 1.2/año & Explotación intensa \\
\hline 2.0/año & Tasa de mortalidad alta \\
\hline
\end{tabular}

Fuente Sparre \& Venema, 1997

Como se observa a pesar de que la tasa de explotación es mediana, la misma se encuentra muy cerca de convertirse a una tasa de explotación intensa, esto se debe a que según Sparre \& Venema (1997), mientras menor es la talla media de captura, mayor será la mortalidad por pesca, en la caso de las redes de tres pulgadas la mortalidad total (Z) fue de 0.49 (límite de confianza de $95 \%$ ), lo indica que esta medida en la luz malla en el arte de pesca produce un nivel de explotación leve. 
El análisis de cohorte de Jones basado en tallas, para la estimación de la mortalidad de pesca $(F)$, implica varios años de datos, sin embargo los datos de un año pueden aplicarse a manera de una seudo cohorte, que consiste en el conjunto de individuos presentes en la población durante un año determinado (Sparre \& Venema, 1997).

Concordando con los resultados de la mortalidad total para redes de dos pulgadas y la distribución de frecuencia de tallas; el análisis de cohorte de Jones para esta especie, demuestra de manera preliminar que se encuentra plenamente explotada y para redes de tres pulgadas de manera preliminar se indica que el recurso todavía no alcanza el nivel de plena explotación y que si esta pesca no se empieza a manejar sosteniblemente, pronto se convertirá en una pesquería sobreexplotada.

La selectividad de las redes de dos pulgadas ocasiona la captura de juveniles; como consecuencia estas redes no permiten que la población de peces se reproduzcan (como se observa en la distribución de frecuencia de tallas). En contraparte la red de tres pulgadas permite la captura de individuos adultos.

Otro estudio (López, 2008), desarrollado en la costa caribeña de Honduras señalo también al Lutjanus synagris (calale) como la especie más importante en la pesca realizada el mar, por los pescadores en el Refugio de Vida Silvestre Barras de Cuero y Salado, alcanzando el nivel de plenamente explotada, dada la importancia de esta especie se debe contemplar medidas técnicas para su manejo sostenible.

\section{CONCLUSIONES}

1. La pesca artesanal es una de las actividades significativas para las comunidades de la Bahía de Tela, no solo por las ventajas que implica realizar esta activad (independencia, nutrición y seguridad alimentaria) pero también, debido a la falta de alternativas económicas y empleo en el municipio de Tela, la pesca es una fuente de ingreso permanente para los pescadores.

2. La pesca artesanal en la Bahía de Tela tiene como objetivos especies que son carnívoras de segundo orden es decir que su dieta alimenticia consiste de invertebrados bentónicos pero también consumen peces. Esto indica que la pesquería en Tela se encuentra en un estado crucial, ya que aún hay un porcentaje de especies que pertenecen a niveles altos en la cadena trófica; sin embargo si se sigue explotando sin un apropiado manejo, es posible que empiece a disminuir y aumente la 
explotación de carnívoros de primer orden y luego herbívoros; afectando la salud del ecosistema y la pesquería.

3. La pesca practicada en Tela es una pesquería dirigida. Sin embargo, este tipo de pesquería sometida a presiones antropogénicas (como las actuales) puede convertirse en una pesquería oportunista, ocasionando una fuerte explotación en las especies y ecosistemas. Las poblaciones de los peces comerciales de alto nivel trófico (como meros y pargos) se han visto fuertemente afectadas por presión pesquera.

4. La participación de la comunidad en el manejo de las pesquerías artesanales, es clave para la planificación y ordenamiento pesquero (ICLARM, 1994). Se propone por consiguiente un co-manejo con la ONG encargada del área protegida. La gestión comunitaria (co-manejo) en la conservación de los recursos pesqueros, requiere de grandes esfuerzos en concienciación y capacitación en organización, los pescadores deben apropiar la idea y saber que protegen "su recurso". Los esfuerzos en concienciación deben orientarse en el Código de Conducta para la Pesca Artesanal propuesto por la FAO.

5. La ausencia de un sistema de regulación al acceso de los recursos pesqueros, ha demostrado ser fuente de sobreexplotación (Berthou, et al, 2001). Por consiguiente, se debe continuar con la extensión del carnet de identificación de pescador. Esta actividad también constituye la validación de los derechos de pesca, necesaria para la promover la autorregulación, que conlleva el co-manejo de los recursos pesqueros.

\section{AGRADECIMIENTOS}

A todos pescadores y familias de pescadores que gentilmente colaboraron y confiaron en el estudio. De las misma manera al Comité Técnico de Tela y a las para técnicas comunitarias que mostraron siempre animosidad, dinamismo y compromiso.

\section{BIBLIOGRAFÍA}

Burke, L., \& Maidens, J. (2005). Arrecifes en peligro en el Caribe. Sin Ciudad Sin 
EditorialCiudad: Editorial

Berthou, P. et al. (2001). Diagnóstico de la pesca artesanal de las Islas de la Bahía. Secretaría de Turismo, Gobierno de Honduras. Islas de la Bahía: Editorial Sin Editorial. $195 \mathrm{p}$.

Cervigón, F. et al. (1992). Guía de campo de las especies comerciales marinas y de aguas salobres de la Costa Septentrional de Sur América. FAO. Roma: Editorial Sin Editorial. $513 p$.

FAO (Ed.). (1996). Recursos marinos vivos y su desarrollo sostenible: perspectivas institucionales y medioambientales: Documento Técnico de Pesca. No. 353. Roma, Italia: FAO. 191p.

FAO. (2010). La Ordenación Pesquera. Orientaciones técnicas para la pesca responsable. Supl. 2, Add. 2. Roma, Italia: Organización de las Naciones Unidas para la Agricultura y la Alimentación.

FAO. (2014). El estado mundial de la pesca y la acuicultura. Roma, Italia: 224 pp.

Gulland, J. y A. Rosenberg, (1992). Examen de los métodos que se basan en la talla para evaluar las poblaciones de peces. Londres: FAO, $114 \mathrm{p}$.

ICLARM,(1994). Community management and common property of coastal fisheries in Asia and the pacific: Concept, methods and experiences. Makatl, Metro Manila, Philippines. International Center for Living Aquatic Resources Management. 189 López, E. A. 2007. Diagnóstico de la pesca artesanal en el Refugio de Vida Silvestre Cuero y Salado. Informe de consultoría FUCSA-WWF. 70 pp.

Sparre, P. \& Venema S.C. (1997). Introducción a la Evaluación de Recursos Pesqueros Tropicales. Parte 1. Manual. FAO Documento Técnico de Pesca. № 306.1 Rev. 2: 420 pág. 48.

PROLANSATE, (2000). La voz de los pescadores de la Costa Atlántica de Honduras. Ciudad de Guatemala: Print Studio. 44 p.

WWF. (2006). Mejores prácticas de pesca en arrecifes coralinos. Guía para la colecta de información que apoye el Manejo de Pesquerías Basado en Ecosistemas. WWF México/Centroamérica. $81 \mathrm{p}$. 